\title{
QUALITY AND RELIABILITY IN HYBRID CIRCUITS
}

\author{
R. F. READ \\ Ministry of Defence, EQD., Golf Rd., Bromley, Kent, U.K.
}

(Received February 2, 1980)

\begin{abstract}
The quality and reliability of hybrid circuits is examined, with particular reference to the BS9000/CECC systems. It is shown that the capability approval approach developed within the BS9000/CECC systems is appropriate to hybrid circuits where only small quantities of each circuit are produced and the steps involved in obtaining capability approval are discussed.
\end{abstract}

\section{INTRODUCTION}

Users of hybrid circuits expect some degree of quality from their suppliers, but how do they quantify their requirements and how do they go about obtaining them? I suspect there are as many views on this subject as there are users.

Quality is not something that can simply be added to the component at will. No matter how many tests the procurement specification invokes, the quality of the component has been established during manufacture and no amount of testing is going to change it. The quality can be assessed or measured and decisions regarding the component's ability to satisfy a particular requirement can be made as a result.

Therefore if we wish to influence the quality of components we must attempt to do it at the manufacturing stage and if we wish to have confidence that the required quality is being achieved, it must be demonstrated that the manufacturing environment is capable of producing it before we commit components to expensive quality assurance testing.

\section{THE BS9000/CECC APPROACH TO QUALITY}

BS9000 is the national system operating in the UK and is harmonized with the European CECC system. Both are based on the surveillance of manufacturers by an independent organisation, the National Supervising Inspectorate.

Each of these systems provides for the following basic requirements:
1) The appraisal and subsequent approval of the manufacturer's organisation and facilities with respect to quality.

2) The Qualification Approval of individual component types to detail specifications or for certain custom built components, e.g. hybrid integrated circuits, the Capability Approval of a particular technology.

3) On going quality conformance inspection.

4) Independent surveillance of the manufacturer's activities by the National Supervising Inspectorate.

5) Certified Test Records of measured performance.

In the UK the National Supervising Inspectorate is involved in all aspects of implementing the BS9000 system and its activities can be broken down into 3 main areas of involvement:

a) Participation in the work of technical committees in the preparation of the main technical (Generic) specifications of the system. The NSI also has a responsibility for the checking and acceptance of detail specifications for individual component types written by component manufacturers.

b) The appraisal of manufacturers, distributors and test laboratories wishing to operate within the system, to ensure that their organisation, facilities and working methods are satisfactory. They also carry out regular surveillance thereafter to ensure that the approval is being adequately maintained.

c) The NSI carry out regular independent audit testing of components manufactured within the system. They use their own laboratories for this purpose using the results of this testing and any subsequent fault analysis to feed back any necessary 
corrective actions, either to the specification system or to the manufacturer's activities.

\section{CAPABILITY APPROVAL FOR HYBRID CIRCUITS}

One of the main difficulties in obtaining assurance of quality for custom-built hybrid circuits using conventional end product assessment procedures, lies in the low volume non-continuous production situation of most hybrid circuit manufacturers. This means that there are seldom sufficient quantities of any one circuit being produced for a long enough period of time for economic statistical sampling to be meaningful.

Additionally each customer order usually involves a new design and therefore the design element becomes a normal part of the hybrid circuit manufacturer's operation.

It is for these reasons that the BS9000/CECC systems developed the new capability approval approach to quality assurance of the custom built components which shifts the emphasis of quality assessment from end product to manufacturing process.

The concept of capability approval is based on a precise definition of the boundaries of a capability, a disciplined design and manufacturing activity, a demonstration that the capability is viable and the subsequent maintenance of the capability by ongoing assessment. This system then sets the scene for quality to a defined standard in manufacture and allows potential customers to negotiate their performance requirements with the hybrid circuit manufacturer in the knowledge that these standards will be applied automatically. The basic requirements for capability approval for hybrid circuits are as follows:

1) The manufacturer is required to provide a description of his capability indicating clearly the technical boundaries and limiting conditions. This takes the form of a capability manual which, when agreed, becomes the yardstick against which he is initially assessed and subsequently monitored. The key points to be covered in the capability manual are:

a) Materials and pieceparts - the manual must identify satisfactory purchase specifications for all critical materials and must describe the methods used to verify the suitability of materials for production. b) Design data and layout rules - the manual must make reference to the detailed rules used in the design and layout of circuits. For example, for thick film hybrid circuits the detailed rules giving appropriate minimum and maximum values must cover conductor tracks, dielectrics and crossovers, resistors, glaze and/or other protection when used, pattern registration, add-on components and thermal characteristics.

c) Flow charts - the manual must include detailed flow charts showing all stages of manufacture and identifying process specifications, working instructions and quality control checks.

d) Capability qualifying circuits - the manual must list the test pieces and or circuits which are to be used to demonstrate the viability of the declared capability. These CQC's must be designed to the declared design rules and collectively must cover all the identified boundaries of the capability.

2) Once the content of the capability manual has been agreed an appraisal takes place in which the NSI check that the procedures etc. laid down in the manual are in fact implemented and are satisfactory.

3) The manufacturer must then agree a test programme with the NSI to demonstrate the viability of his declared capability. This test programme will use as test vehicles the capability qualifying circuits listed in the manual.

These circuits must be designed using the agreed design rules and should include the critical limits of those rules. For example, for thick film hybrids the following features will normally be covered:

a) Pads for adhesion tests

b) Conductors of min. width at min. spacing

c) Resistors of smallest and largest aspect ratio covering the range of resistor inks

d) Largest size resistor dissipating max. rated power

e) Two identical trimmed resistors to demonstrate matching and tracking

f) Smallest size resistor

g) A dielectric crossover

h) A solder mounted chip capacitor

i) An epoxy mounted power transistor chip with wire bonds.

j) An epoxy mounted IC chip (preferably MOS) with wire bonds.

The CQC's must be packaged in representative exarples of the range of packages offered by the manufacturer and in addition to meeting the test requirements for the design characteristics must satisfy a schedule of environmental and endurance 
tests. In general these are standard tests such as solderability, rapid change of temperature, damp heat, vibration, shock etc., and the endurance covers both a 160 -hour and a 2000 -hour period.

4) On completion of the demonstration a satisfactory report results in approval being granted for the manufacturers capability within the scope of his capability manual.

From this point in time the manufacturer is bound by the BS9000/CECC rules governing changes. This means that he cannot make changes to either his capability manual or his manufacturing processes without adequate demonstration to the NSI that the proposed changes do not reduce the quality of components. Changes to extend the scope of the capability can, of course, be recognised by the system after the proposed extension has been properly assessed.

5) In order to maintain his approval the manufacturer must carry out tests on customer circuits in accordance with the detail specification negotiated with the customer, and in addition must submit CQC's to:

a) A schedule of short-term environmental and endurance tests, on a lot-by-lot basis

b) A schedule of medium-term environmental and endurance tests on a periodic basis, normally every 3 months, the endurance test is for a 2000 hour period.

c) A schedule of long-term environmental and endurance tests, on a periodic basis, normally every year, the endurance test is for an 8000-hour period. These test schedules are specified in the generic specification for custom built integrated circuits.

6) The manufacturer must accumulate test data from maintenance of capability approval testing of CQC's to compile certified test records every 6 months. The certified test record must give test results for the current 6-month and 3-year periods and be available to bona fide customers on request.

\section{CONCLUSION}

The BS9000/CECC system provides a cost effective solution to many of the problems of controlling component activity. By establishing common terminology, appropriate assessment methods and specifications it permits economy of scale in the manufacturing industry through the aggregation of customer requirements. Providing an effective quality assurance support for the equipment industry BS9000/CECC reduces overhead costs and creates an objective data base for circuit and system designers.

\section{ACKNOWLEDGEMENTS}

Permission to re-publish this paper is gratefully acknowledged. The paper is copyright $\odot$, Controller HMSO, London, 1979. 

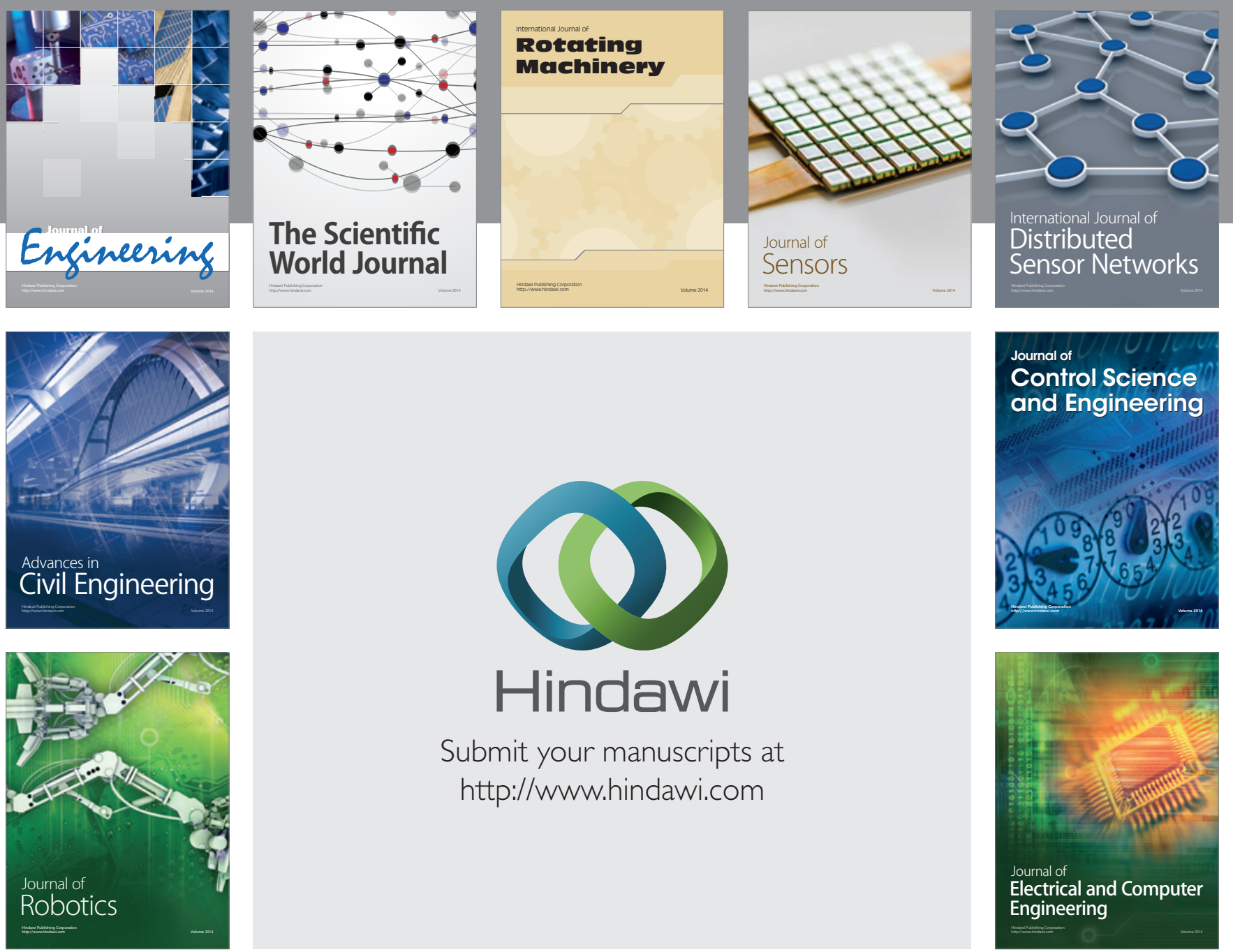

Submit your manuscripts at

http://www.hindawi.com
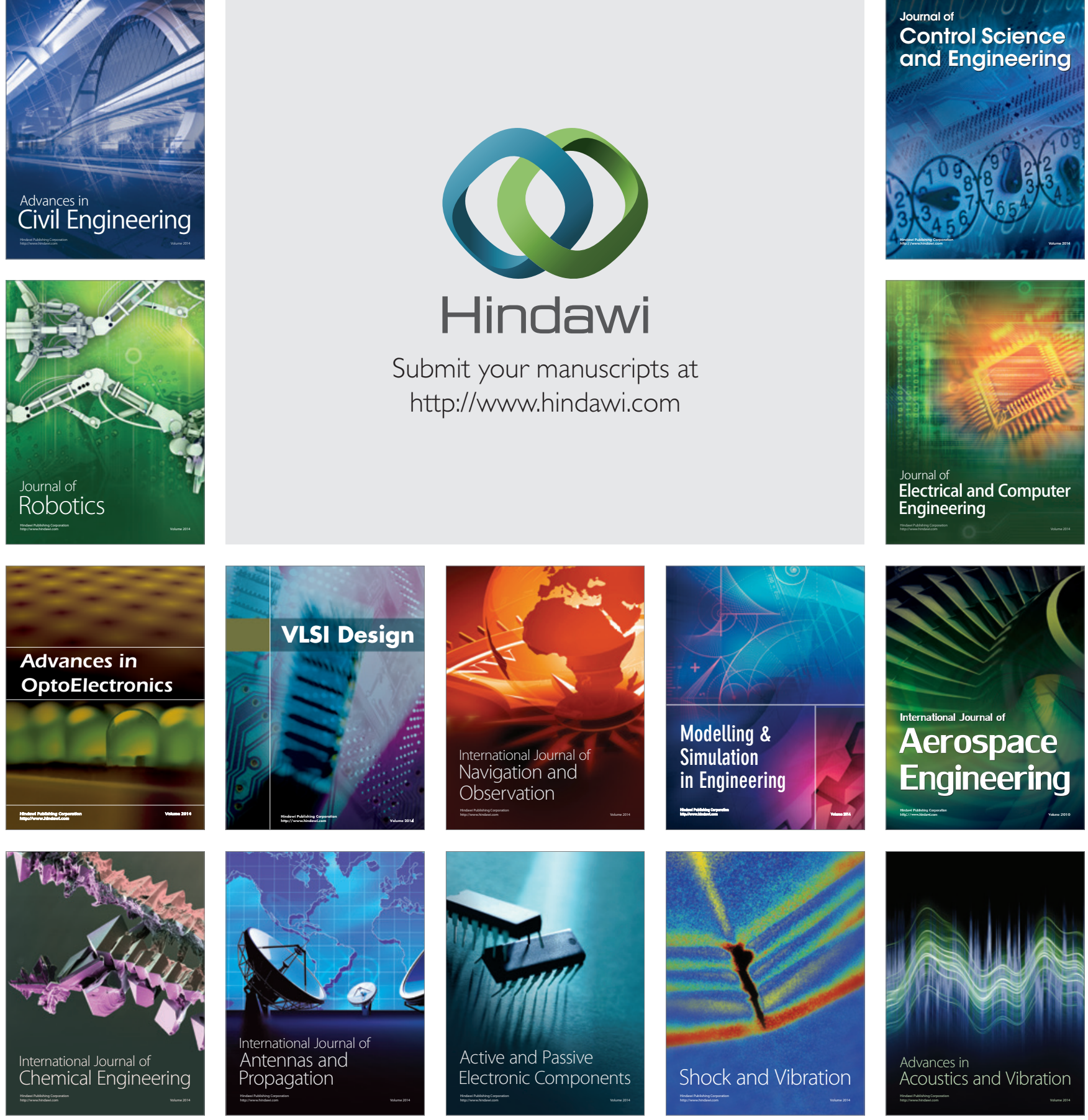